\title{
On the absence of wind bow-shocks around OB-runaway stars: Probing the physical conditions of the interstellar medium
}

\author{
F. Huthoff ${ }^{1}$ and L. Kaper ${ }^{1}$
}

\begin{abstract}
Astronomical Institute "Anton Pannekoek" and Center for High-Energy Astrophysics, University of Amsterdam, Kruislaan 403, 1098 SJ Amsterdam, The Netherlands

Received 21 September 2001 / Accepted 11 December 2001

Abstract. High-resolution IRAS maps are used to search for the presence of stellar-wind bow-shocks around high-mass X-ray binaries (HMXBs). Their high space velocities, recently confirmed with Hipparcos observations, combined with their strong stellar winds should result in the formation of wind bow-shocks. Except for the already known bow-shock around Vela X-1 (Kaper et al. 1997), we do not find convincing evidence for a bow-shock around any of the other HMXBs. Also in the case of (supposedly single) OB-runaway stars, only a minority appears to be associated with a bow-shock (Van Buren et al. 1995). We investigate why wind bow-shocks are not detected for the majority of these OB-runaway systems: is this due to the IRAS sensitivity, the system's space velocity, the stellar-wind properties, or the height above the galactic plane? It turns out that none of these suggested causes can explain the low detection rate $(\sim 40 \%)$. We propose that the conditions of the interstellar medium mainly determine whether a wind bow-shock is formed or not. In hot, tenuous media (like inside galactic superbubbles) the sound speed is high $\left(\sim 100 \mathrm{~km} \mathrm{~s}^{-1}\right)$, such that many runaways move at subsonic velocity through a low-density medium, thus preventing the formation of an observable bow-shock. Superbubbles are expected (and observed) around OB associations, where the OB-runaway stars were once born. Turning the argument around, we use the absence (or presence) of wind bow-shocks around OB runaways to probe the physical conditions of the interstellar medium in the solar neighbourhood.
\end{abstract}

Key words. stars: early-type - stars: kinematics - stars: mass loss - ISM: bubbles - ISM: structure X-rays: binaries

\section{Introduction}

OB-runaway stars are massive, OB-type stars with high space velocities. This small $(\sim 20 \%$ of the $\mathrm{O}$ stars, less than $5 \%$ of the early B stars), but significant excess of high-velocity objects suggests a systematical production of fast movers (Feast \& Shuttleworth 1965; Stone 1979). Among the OB runaways, velocities well above $100 \mathrm{~km} \mathrm{~s}^{-1}$ have been observed (Gies \& Bolton 1986; Conlon et al. 1990; Blaauw 1993), which is roughly ten times the average space velocity of "normal" OB stars in the Milky Way. Due to their high velocities, OB-runaways may have travelled great distances since their formation. The criteria used to classify OB stars as "runaways" are (Blaauw 1961): (i) a high space velocity; often a threshold space velocity of $30 \mathrm{~km} \mathrm{~s}^{-1}$ is used, roughly corresponding to three times the typical space velocity of OB-type stars. (ii) The reconstructed path of the runaway should start in a "parent" OB association. Since the definition by Blaauw, the term $O B$ runaway has often been used to include all of the high-velocity OB stars, irrespective of the identification of a parent OB-association. A large distance above

Send offprint requests to: L. Kaper,

e-mail: lexk@science.uva.nl the galactic plane can also be used as an indication for the runaway-nature of a massive star (e.g. Van Oijen 1987).

The two most popular scenarios for the production of runaways seem to operate at roughly the same rate (Hoogerwerf et al. 2001) and are both capable of producing runaways with velocities up to $200 \mathrm{~km} \mathrm{~s}^{-1}$ (PortegiesZwart 2000; Leonard 1995): (i) the binary supernova scenario (Blaauw 1961; Zwicky 1957), where a massive star in a binary receives a high space velocity after the supernova explosion of its (initially) more massive companion; (ii) the cluster ejection scenario (Poveda et al. 1967), where dynamical interactions in a compact cluster result in the ejection of one or more of its members. Especially encounters between binary systems are effective in producing high-velocity objects (e.g. Mikkola 1983).

The current version of the binary supernova scenario includes a phase of mass transfer (Van den Heuvel \& Heise 1972): when the initially most massive star fills its Roche lobe (e.g. when becoming a supergiant) mass is transfered to its companion which eventually will become the most massive star in the system. As a consequence, the system has a high probability to remain bound after the supernova explosion of the initially most massive star (Boersma 1961). If it remains bound, the binary, now consisting of 
Table 1. Prediction of the angular separation between wind bow-shock and HMXB. The HMXB peculiar velocity (i.e. corrected for differential galactic rotation and peculiar solar motion) in the radial and tangential direction is listed in Cols. 6 and 7 , respectively; the resulting space velocity $v_{\text {space }}$ is given in Col. 8 . The first five columns list the identification of the $\mathrm{X}$-ray source, the name and spectral type of the OB companion (if known), the distance $r$, and the height $z$ above the galactic plane. The predicted (and observed, final column) angular distance between OB star and wind bow-shock are given in the last columns. For the HMXBs that have not been observed with Hipparcos $\left(v_{\tan }\right)_{\mathrm{pec}}=50 \mathrm{~km} \mathrm{~s}^{-1}$ has been adopted. If a question mark is added, infrared emission is detected, but no clear arclike structure could be resolved. The prediction of the stand-off distance is based on stellar-wind parameters taken from Puls et al. (1996) and Kaper et al. (1998); for Be stars it was assumed that $\dot{M}=10^{-8} M_{\odot} \mathrm{yr}^{-1}$ and $v_{\infty}=250 \mathrm{~km} \mathrm{~s}^{-1}$.

\begin{tabular}{llllllll|lc|cc}
\hline Object & & Spectral Type & $\begin{array}{r}r \\
(\mathrm{kpc})\end{array}$ & $\begin{array}{r}z \\
(\mathrm{pc})\end{array}$ & $\begin{array}{c}\left(v_{\mathrm{rad}}\right)_{\mathrm{pec}} \\
\left(\mathrm{km} \mathrm{s}^{-1}\right)\end{array}$ & $\begin{array}{c}\left(v_{\text {tan }}\right)_{\mathrm{pec}} \\
\left(\mathrm{km} \mathrm{s}^{-1}\right)\end{array}$ & $\begin{array}{c}v_{\text {space }} \\
\left(\mathrm{km} \mathrm{s}^{-1}\right)\end{array}$ & $\begin{array}{c}d_{\text {shock }}^{\text {pred }} \\
(\mathrm{pc})\end{array}$ & $\begin{array}{c}\delta_{\text {shock }}^{\text {pred }} \\
\left({ }^{\prime}\right)\end{array}$ & $\begin{array}{c}\text { bow-shock? } \\
\delta_{\text {shock }} \\
\left({ }^{\prime}\right)\end{array}$ \\
\hline $0114+650$ & & B0.5 Ib & 3.8 & 171 & -9.6 & 29.4 & 31.0 & 2.6 & 2.4 & no & \\
$1223-624$ & GX301-2 & B1.5 Ia & 5.0 & -3.0 & -1.6 & 50 & 50 & 2.8 & 1.9 & $?$ & $1.5(?)$ \\
$1700-377$ & HD 153919 & O6.5 Iaf & 1.7 & 65 & -42.0 & 57.3 & 71.0 & 3.1 & 6.3 & $?$ & $8.0(?)$ \\
$1907+097$ & & B0.5 Ib & 4.0 & 37 & & 50 & 50 & 0.6 & 0.5 & $?$ & $0.5(?)$ \\
$1538-522$ & QV Nor & B0 Iab & 5.5 & 206 & -81.9 & 50 & 96 & 1.9 & 1.2 & no & no \\
$1119-603$ & Cen X-3 & O6.5 II-III & 8.0 & 47 & 16.3 & 50 & 53 & 1.7 & 0.7 & no \\
$1956+350$ & Cyg X-1 & O9.5 Iab & 2.5 & 135 & -10.9 & 41.4 & 42.8 & 5.0 & 6.9 & yes & 1.0 \\
$0900-403$ & Vela X-1 & B0.5 Ib & 1.8 & 123 & -22.3 & 38.5 & 44.5 & 1.4 & 2.7 & no \\
$0236+610$ & V615 Cas & B0e & 2.0 & 42 & -30.2 & 22.5 & 37.7 & .14 & .24 & no \\
$0535+262$ & V725 Tau & O9.7e II & 2.0 & -90 & -41.5 & 57.5 & 70.9 & .07 & .12 & no \\
$0352+309$ & X Per & O9e III-IV & 0.8 & -235 & -32.0 & 13.4 & 34.7 & .51 & 2.2 & no \\
\hline
\end{tabular}

a massive, rejuvenated main-sequence star and a compact remnant (a neutron star or a black hole), will travel through space with a high velocity. As soon as the massive star becomes a supergiant, material from its dense stellar wind (or through Roche-lobe overflow) is intercepted by the gravitational field of the compact star and accretes, powering a strong X-ray source: a high-mass X-ray binary (HMXB). This scenario thus predicts that all HMXBs are runaway objects. Recently, this prediction has been confirmed with Hipparcos observations (Chevalier \& Ilovaisky 1997; Kaper et al. 1999). This also suggests that several supposedly single OB runaways might have a, so far undetected, compact companion. For a review on HMXBs and OB-runaway stars, see Kaper (2001).

The high space velocity and powerful stellar wind of the OB-runaway star will give rise to a strong interaction with the ambient medium. In the case of supersonic movement, a wind bow-shock is formed (Baranov et al. 1971). The wind bow-shock accumulates gas and dust from the interstellar medium which is heated by the ultraviolet radiation field of the $\mathrm{OB}$ star. The heated dust subsequently radiates at infrared wavelengths (Draine \& Lee 1985) and the shocked gas becomes visible in strong optical emission lines like $\mathrm{H} \alpha$ and [O III] 4959, $5007 \AA$ (e.g. Kaper et al. 1997). Data obtained with the InfraRed Astronomical Satellite (IRAS) has been used to search for the presence of wind bow-shocks around OB runaways by Van Buren \& McCray (1988) and Van Buren et al. (1995). They detected excess infrared $(60 \mu \mathrm{m})$ emission for about $30 \%$ of the $188 \mathrm{OB}$ stars in their sample. For a significant fraction prominent arc-like features could be resolved using high-resolution maps (Noriega-Crespo et al. 1997). When spatially resolved, the shape of the bowshock indicates the direction of motion of the system. The stand-off distance of the shock provides a constraint on the space velocity, the stellar-wind parameters, and the density of the ambient medium.

In this study we investigate whether wind bow-shocks are as common for HMXBs as for (single) OB-runaway stars, by searching for their infrared emission in highresolution maps produced from data obtained with the IRAS satellite. We find that also in the case of HMXBs only a minority form a wind bow-shock. We investigate whether the detection of a bow-shock relates to (i) the kinematical properties of the runaways; (ii) the height above the galactic plane; or (iii) the spectral type of the OB runaway. The remaining option, namely that the physical conditions of the ambient medium mainly determine whether a wind bow-shock is formed, seems to be the most likely one. If true, this provides the opportunity to use OB runaways as probes of the physical conditions of the interstellar medium.

\section{A search for bow-shocks around HMXBs}

In our search for the presence of wind bow-shocks around high-mass X-ray binaries we selected a number of systems for which the conditions for the formation of a bow-shock 


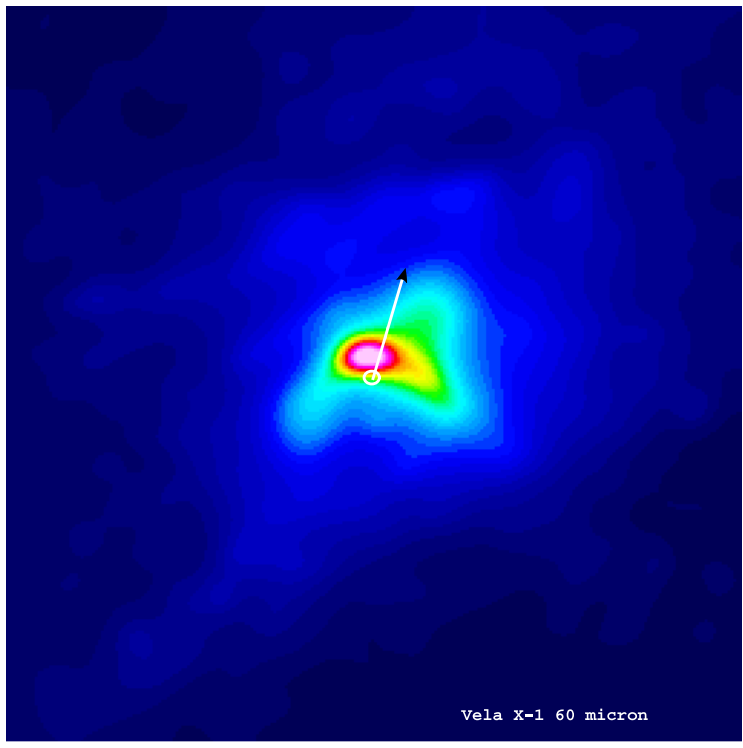

Fig. 1. HIRAS image of the circumstellar environment of Vela X-1 at $60 \mu \mathrm{m}$. The arc-like structure of a wind bow-shock is clearly resolved. The arrow indicates the direction of the system's space velocity. East is to the left and North is up; the image size is $0.5^{\circ} \times 0.5^{\circ}$. Infrared emission of the bow-shock is also detected in the other three IRAS bands.

seem favourable: a large space velocity and an OB companion with a strong stellar wind and a high luminosity. In practice this means that our sample consists mainly of systems hosting an OB supergiant (cf. Kaper 1998). We also included a few Be/X-ray binaries (with early-type OBe companion). The HMXB sample is listed in Table 1. To determine the size of the area on the sky to inspect for the presence of a bow-shock, we estimated the angular distance from the star at which a bow-shock should form. The stand-off distance $\left(d_{\mathrm{s}}\right)$ is set by the balance in ram pressure between the stellar wind and the ambient medium (cf. Wilkin 1996). Using typical values for the wind massloss rate $\dot{M}$ (in $M_{\odot} \mathrm{yr}^{-1}$ ), wind terminal velocity $v_{\infty}$ and space velocity $v_{\star}\left(\right.$ in $\mathrm{km} \mathrm{s}^{-1}$ ), and the density $\left(\right.$ in $\mathrm{cm}^{-3}$ ) of the ambient medium $n\left(=\rho / \mu \mathrm{m}_{\mathrm{H}}\right)$, the stand-off distance can be written as:

$$
\begin{aligned}
d_{\mathrm{s}}= & \frac{0.9 \mathrm{pc}}{\left(v_{\star} / 50 \mathrm{~km} \mathrm{~s}^{-1}\right)} \\
& \times \sqrt{\frac{\left(\dot{M} / 10^{-6} M_{\odot} / \mathrm{yr}\right) \times\left(v_{\infty} / 10^{3} \mathrm{~km} \mathrm{~s}^{-1}\right)}{n / \mathrm{cm}^{-3}}} .
\end{aligned}
$$

We calculated $d_{\mathrm{s}}$ for the systems in our sample (Table 1 ). Stellar-wind parameters were taken from Puls et al. (1996) and Kaper et al. (1998). The space velocity is obtained from the observed Hipparcos proper motion and radial velocity, corrected for differential galactic rotation and peculiar solar motion (cf. Moffat et al. 1998). The interstellar medium density was computed with the mean density model of Dickey \& Lockman (1990). The typical area on the sky that we inspected for the presence of a bow-shock is $0.5^{\circ} \times 0.5^{\circ}$.

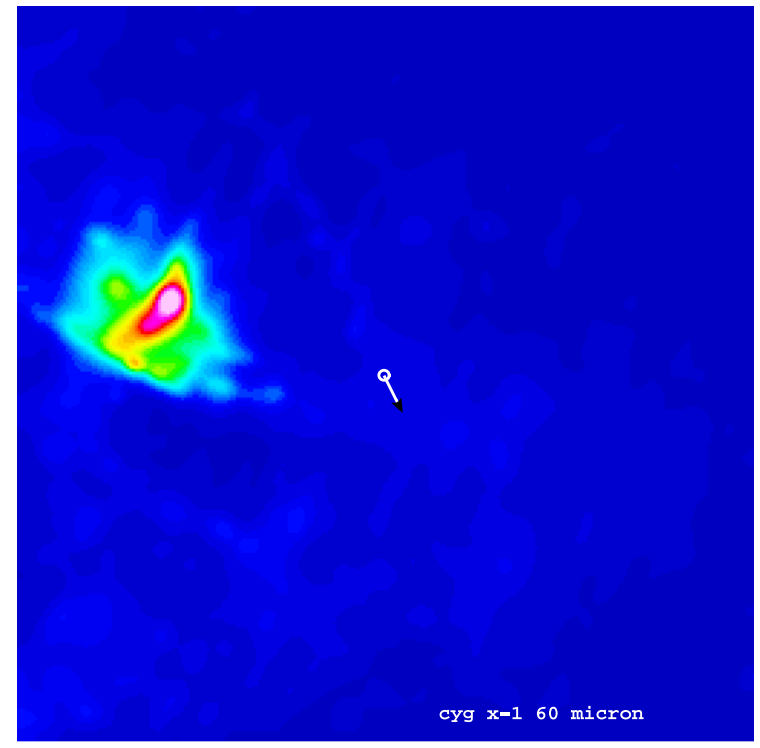

Fig. 2. HIRAS image of the environment of Cygnus X-1 at $60 \mu \mathrm{m}$. The feature located roughly $20^{\prime}$ east of Cyg X-1 is part of a larger filament that outlines the Cygnus $\mathrm{X}$ region (Cash et al. 1980): a large X-ray emitting region possibly heated by numerous supernovae and winds from massive stars. East is to the left and North is up, the image size is $1.0^{\circ} \times 1.0^{\circ}$.

\subsection{HIRAS maps of the HMXB environment}

We used the program HIRAS (Bontekoe et al. 1994) to produce high-resolution IRAS maps of the circumstellar environment of the HMXB sample, to inspect the area for the presence of wind bow-shocks. The IRAS satellite scanned almost the whole infrared sky in four wavelength bands $(12,25,60$, and $100 \mu \mathrm{m})$ in strips of about $6^{\prime}$ wide. The HIRAS software takes advantage of the fact that a given part of the sky has been observed several times, resulting in strips that partly overlap. Combining these observations then yields a significant enhancement in spatial resolution. For details of the HIRAS-algorithm and the used method we refer to Bontekoe et al. (1994). The resolution of the images depends on the local sampling pattern and, therefore, varies with position on the sky; in our HIRAS images it typically is in the range from $0.4^{\prime}$ to $1.7^{\prime}$.

For each system HIRAS images were prepared in the four IRAS bands. A wind bow-shock has been detected in a narrow-band $\mathrm{H} \alpha$ image of the field surrounding the B-supergiant HD77581, the companion of the X-ray pulsar Vela X-1 (Kaper et al. 1997). The bow-shock is clearly present in the HIRAS images too. Figure 1 shows a HIRAS image of Vela X-1 at $60 \mu \mathrm{m}$, with the direction of its peculiar motion indicated. We prepared images in the other three IRAS bands (12, 25 and $100 \mu \mathrm{m}$, not shown here), but the bow-shock (and at 60 and $100 \mu \mathrm{m}$ the $\mathrm{H}$ II region surrounding the system) is detected in all four bands. The arc-like structure of the bow-shock is resolved at 25 and $60 \mu \mathrm{m}$. Images obtained with ISOCAM onboard the Infrared Space Observatory confirm the detection at 6 and $12 \mu \mathrm{m}$, and reveal the fine structure present in the $\mathrm{H} \alpha$ image (Kaper et al. 1998). The symmetry axis of the 


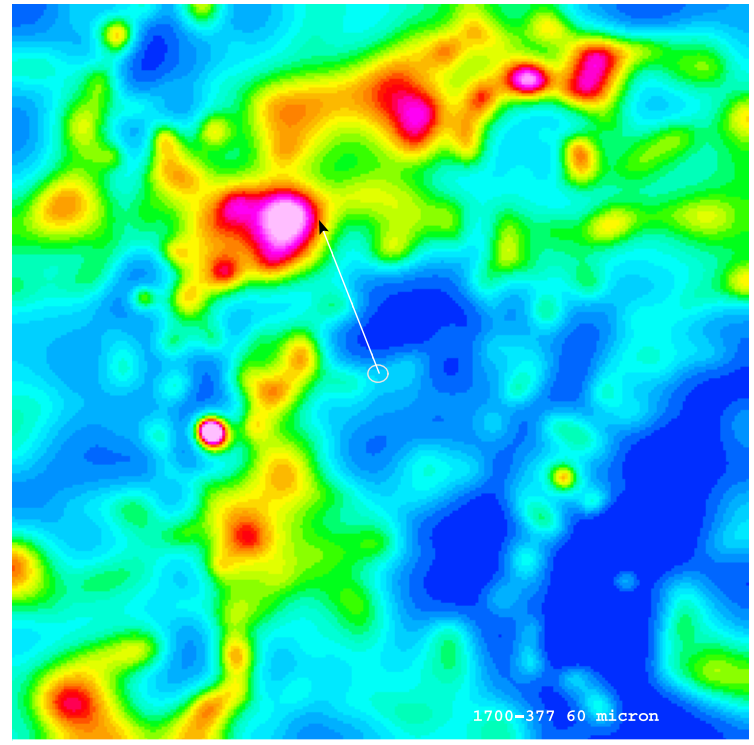

Fig. 3. HIRAS image of the area on the sky centered at $1700-$ 377 at $60 \mu \mathrm{m}$. Many infrared sources are located in the vicinity of 1700-377, making it difficult to identify a possible wind bowshock feature associated with this HMXB. East is to the left and North is up, the image size is $0.5^{\circ} \times 0.5^{\circ}$.

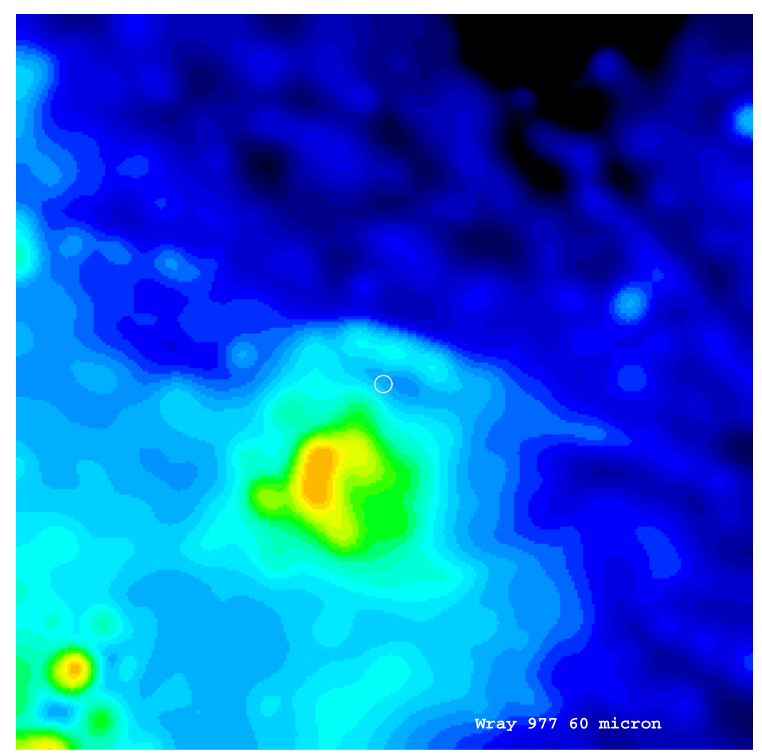

Fig. 4. HIRAS image of GX301-2 at $60 \mu \mathrm{m}$. Infrared emission is detected which could be attributable to a wind bow-shock. However, this is a complicated area on the sky, many other infrared sources are located within the field of view. East is to the left and North is up, the image has a size of $0.5^{\circ} \times 0.5^{\circ}$.

bow-shock is parallel to the system's direction of motion; its space velocity is $45 \mathrm{~km} \mathrm{~s}^{-1}$ (based on Hipparcos observations; note that Kaper et al. 1997 wrongly quote $90 \mathrm{~km} \mathrm{~s}^{-1}$ ). Moreover, the infrared emission of the shockfront is coincident with the region producing $\mathrm{H} \alpha$ emission.

Based on the size of the $\mathrm{H}$ II region surrounding HD77581, Kaper et al. (1997) derive that the number density of the ambient medium is $\sim 10 \mathrm{~cm}^{-3}$, which is about ten times higher than predicted with the model of

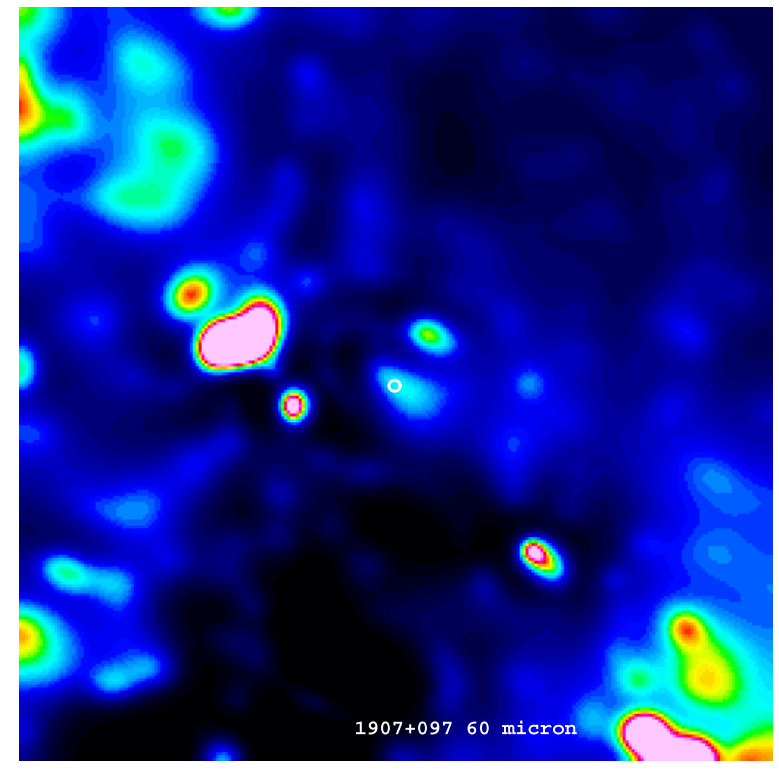

Fig. 5. HIRAS image of the HMXB $1907+097$ at $60 \mu \mathrm{m}$. Possibly this system is producing a wind bow-shock, but this could be a chance superposition. Infrared emission was detected in all four IRAS bands. Note that the proper motion of $1907+097$ is not known. East is to the left and North is up, the image has a size of $0.5^{\circ} \times 0.5^{\circ}$.

Dickey \& Lockman (1990) and a distance of 120 pc above the galactic plane. The higher density is also consistent with the observed stand-off distance, which is less than predicted (Table 1).

None of the fields we inspected show evidence for arclike structures that can be associated with a wind bowshock around any of the other HMXBs. It is not surprising that the three Be/X-ray binaries, which do not drive strong stellar winds, do not produce a bow-shock. On average, these systems also have lower space velocities (Chevalier \& Ilovaisky 1997); this is consistent with the predictions of models describing the evolution of massive binary systems (Van den Heuvel et al. 2000). However, a few OB-supergiant systems do show some interesting features that are worthwhile discussing. Figures $2-5$ show HIRAS images of some of these systems. The size (and direction) of the depicted arrow is proportional to the peculiar space velocity of the system (the size of the arrow corresponds to the displacement over the next 60,000 years).

In three cases it remains unclear whether the observed HMXBs are producing bow-shocks. In the case of 1700-377 and GX301-2 bright objects are present in the field possibly hampering the identification of a bow-shock (Figs. 3 and 4, $60 \mu \mathrm{m}$ images). GX301-2 is surrounded by a faint ring-like structure at an angular distance of $\sim 2^{\prime}$ towards the north. As we do not know the proper motion of the system it is hard to say whether this structure is related to GX301-2. 1907+097 shows some emission in all four IRAS-bands at the location of the source, a bow-shock might be present. At a distance of $\sim 4 \mathrm{kpc}$ the shockfront is expected to have an angular separation of $\sim 0.5^{\prime}$, consistent with what is observed. 
In summary, only one of the HMXBs in our sample is, beyond any doubt, associated with a wind bow-shock. The question remains why the other systems do not produce a (detectable) bow-shock? They are moving at high velocity and have strong stellar winds powering an X-ray source. Unfortunately, our sample is too small to perform a statistical study to determine why wind bow-shocks are absent. The sample of OB runaways studied by Van Buren et al. (1995) is much better suited for such an investigation.

\section{Wind bow-shocks around supposedly single OB-runaway stars}

Van Buren et al. (1995) conducted an extended search for wind bow-shocks around OB runaways. Based on pre-Hipparcos proper-motion and radial-velocity measurements a total of 188 candidate runaway stars was compiled from the catalogs of Garmany et al. (1982), Cruz-Gonzales et al. (1974) and the entire list by Stone (1979). Van Buren et al. (1995) correctly point out that this list should not be considered systematic or complete since it inherits the biases of the input catalogs. Among that sample, 58 candidate bow-shocks were identified based on low-resolution images from the IRAS Sky Survey Atlas (ISSA). In a follow-up study by NoriegaCrespo et al. (1997) the 58 candidate bow-shocks were more closely examined. They analyzed $60 \mu \mathrm{m}$ IRAS highresolution (HiRes) images of these objects that were produced with a maximum correlation algorithm (Fowler \& Aumann 1994), a method similar to the one we discussed in the previous section. Objects were classified as being either (i) bow-shock producing objects, or, (ii) if a shockfront detection was inconlusive but the object showed excess infrared radiation, as excess IR objects. Three objects did not fall in any of these catagories and were rejected (or rather, classified as non-detections).

As mentioned above, Van Buren et al. (1995) concluded that about a third of the 188 OB runaways are associated with a bow-shock, i.e. the majority of the systems do not produce an (observable) wind bow-shock. Our aim is to determine whether the properties of OB runaways with a bow-shock differ from those for which a bow-shock seems to be absent. We investigate the spatial distribution and the kinematical properties of the OB runaways, their galactic height distribution, and their spectral types.

\subsection{Properties of Van Buren's OB-runaway sample}

We checked the Hipparcos database and found that 168 stars from Van Buren's total of 188 candidate runaways are included. We omitted stars with spectral type other than $\mathrm{O}$ or $\mathrm{B}$ and rejected all objects located at distances beyond $4 \mathrm{kpc}$ because of the large uncertainty in proper motion. As a result we were left with a sample of 148 candidate runaways, to which will be refered to as "Van Buren's sample". Radial velocities were taken from the SIMBAD database; for objects for which no radial velocity is measured, the tangential velocity is multiplied by
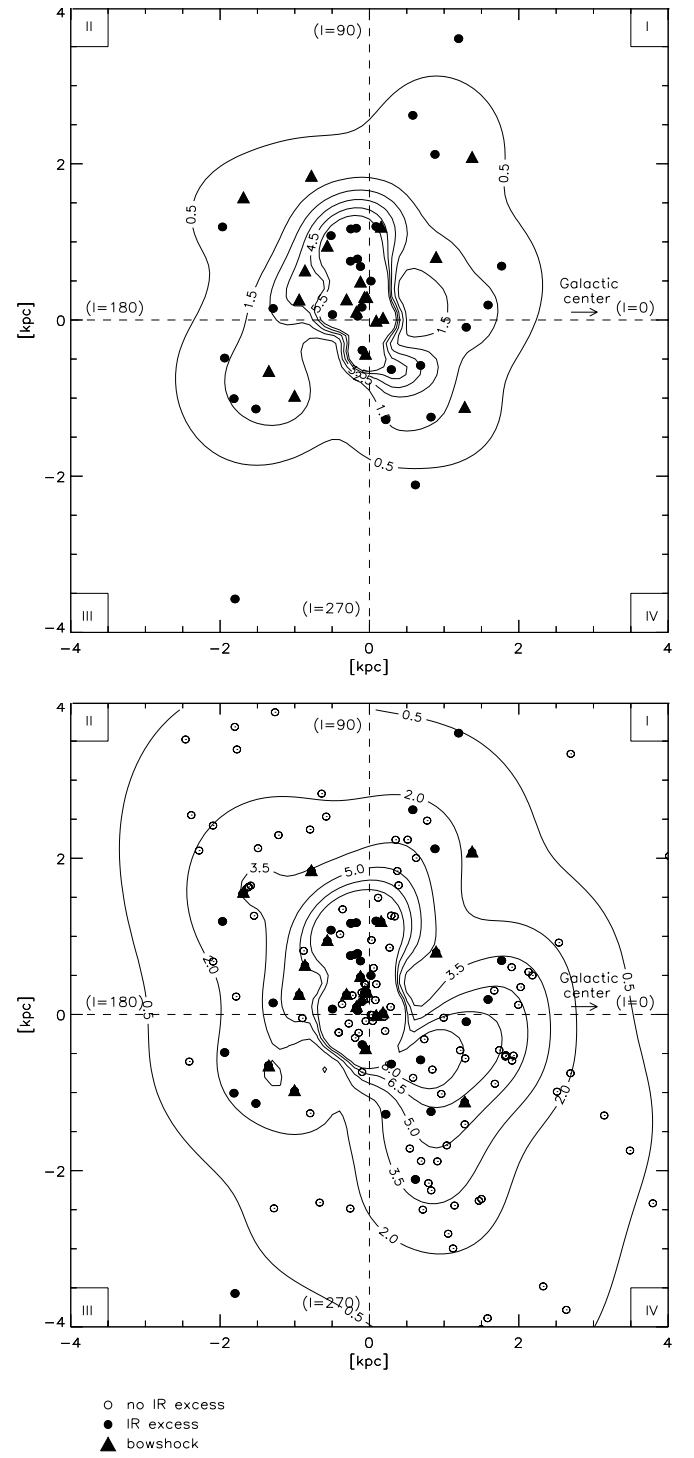

Fig. 6. The spatial distribution of the OB runaways in Van Buren's sample (bottom panel). The Sun is located at the origin of the diagram. The density contours were calculated with the so-called kernel method: each location of a runaway is replaced by a normal distribution with a standard deviation corresponding to $30 \%$ of the heliocentric distance. The spatial distribution of the OB-runaway stars that produce either bow-shocks (filled triangles) or show excess infrared emission (filled circles) is displayed in the top panel. The space density of runaways with a bow-shock decreases significantly with distance, most likely due to the reduced probability of detecting a bow-shock.

a factor $\sqrt{3 / 2}$ when calculating the space velocity. Proper motions and radial velocities were corrected for differential galactic rotation and the peculiar solar motion. We followed the approach described in Moffat et al. (1998) which uses a flat galactic rotation curve.

\subsubsection{Spatial distribution}

The spatial distribution of the OB runaways included in Van Buren's sample (and with a distance less than 
about $4 \mathrm{kpc}$ ) is shown in Fig. 6 . The top panel displays the OB runaways with a detected wind bow-shock (filled triangles) as well as those exhibiting excess infrared emission (filled circles). The density contours were calculated using the binormal kernel method, where the position of each runaway star is replaced by a binormal distribution (i.e. with standard deviation $\sigma_{\mathrm{r}}$ in both $x$ and $y$ ). Subsequently, the convolution of all binormal distributions results in a continuous OB-runaway density distribution in the galactic plane. For instance, let $d_{i}$ be the distance between an arbitrary position $\left(x_{i}, y_{i}\right)$ in the galactic plane and the location $(x, y)$ of an OB runaway:

$d_{i}=\sqrt{\left(x-x_{i}\right)^{2}+\left(y-y_{i}\right)^{2}}$

then the kernel is:

$K_{r}\left(d_{i} / \sigma_{r}\right)=\frac{1}{2 \pi} \mathrm{e}^{-\frac{d_{i}^{2}}{2 \sigma_{r}^{2}}}$

where $\sigma_{\mathrm{r}}$ is an adaptive spread in the kernel function. We chose the value of $\sigma_{\mathrm{r}}$ (the width of the distribution) to depend on the heliocentric distance $(r)$ to the respective runaway:

$\sigma_{r}=a \times r$.

We varied $a$ between 0.1 and 0.5 , resulting in a more continuous distribution with increasing $a$; we adopted $a=0.3$ to produce Fig. 6. Even though the positional accuracy of each runaway is considerably smaller along the tangential direction, $\sigma_{\mathrm{r}}$ is taken to be symmetric in both directions for reasons of simplicity. Adding all individual kernels, the density distribution becomes:

$\rho(x, y)=\sum_{i=1}^{n} \frac{1}{\sigma_{r}^{2}} K_{r}\left(d_{i} / \sigma_{r}\right)$.

Note that each term carries a normalization factor with the dimensions $\mathrm{kpc}^{-2}$. The distribution of $\rho(x, y)$ thus reflects the surface density of OB runaways.

The same method was used to derive the density distribution of bow-shock producing runaways only (top panel Fig. 6). It was assumed that objects that show excess infrared emission are producing wind bow-shocks. Figure 6 indicates that the number of $\mathrm{OB}$ runaways that produce observable bow-shocks drops significantly beyond a distance of $2 \mathrm{kpc}$. The relative abundance of objects showing either a bow-shock or IR-excess remains nearly constant $(\sim 40 \%)$ up to a distance of about $2 \mathrm{kpc}$. Within $0.5,1.0$, and $2.0 \mathrm{kpc}$ we respectively find 13 (out of 33 ), 18 (44), and 39 (98) runaways with a bow-shock; this corresponds to 39,41 , and $40 \%$, respectively. Between 2.0 and $4.0 \mathrm{kpc}$ we encounter a bow-shock for 9 out of 50 runaways (18\%). Therefore, even if the sample becomes less complete at larger distances, the roughly constant fraction of bow-shocks among the runaways up to a distance of $2 \mathrm{kpc}$ suggests that the sampling of the runaway population producing bow-shocks becomes incomplete beyond that distance. This might be due to the reduction in the detection sensitivity of IRAS.

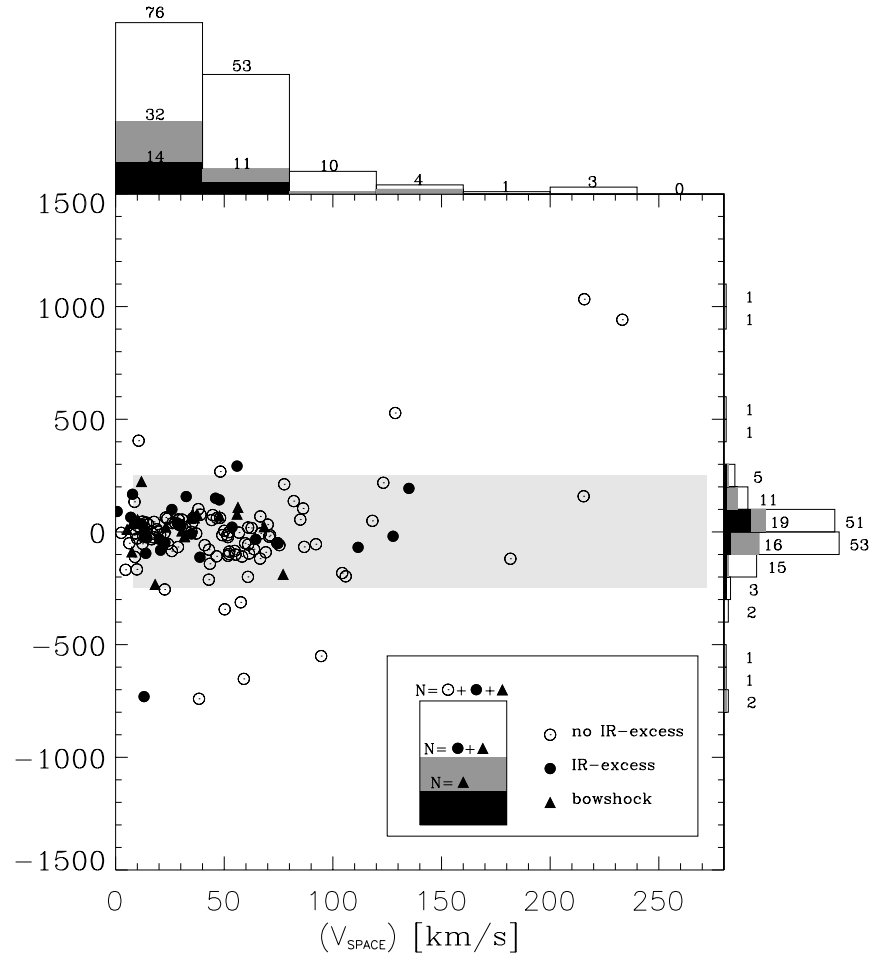

Fig. 7. Space velocity versus distance $z$ to the galactic plane. This graph shows that only a small number of objects with a very high space velocity or with a large $z$ distance produce bow-shocks (the meaning of the symbols is indicated in the legenda). On the other hand, a large fraction of the objects with a space velocity less than $40 \mathrm{~km} \mathrm{~s}^{-1}$ do show a bowshock: $42 \%$ are associated with a bow-shock or show IR excess; at higher velocities this rate is only $22 \%$. The number of objects within a given space-velocity or $z$-distance bin is indicated along the axes. The cumulative number of objects with a bow-shock (black), with IR excess (grey), or none of the two (white) is included.

A short intermezzo: The sensitivity of IRAS is, at best, $\sim 0.2$ Jy at 12,25 , and $60 \mu \mathrm{m}$, and $\sim 1$ Jy at $100 \mu \mathrm{m}$, per $0^{\prime} .25 \times 0^{\prime} .25$ or $0^{\prime} .5 \times 0^{\prime} .5$ pixel at 12 and 25 or 60 and $100 \mu \mathrm{m}$, respectively (Moshir et al. 1989). The highest IRAS flux per pixel measured in the bow-shock associated with Vela X-1 (Fig. 1) is 0.03 (0.01), 0.41 (0.01), 2.59 (0.06), and $1.04(0.21) \mathrm{Jy} / \mathrm{px}$ at $12,25,60$, and $100 \mu \mathrm{m}$, respectively; the value between brackets is the level of the infrared background in that direction. The heliocentric distance to Vela X-1 is $1.8 \mathrm{kpc}$; according to the quoted IRAS sensitivity the bow-shock would still be marginally detectable (only at $60 \mu \mathrm{m}$ ) if the distance to Vela X-1 is increased by about a factor 3 . In terms of integrated flux $(2.8,38,95$, and $34 \mathrm{Jy})$ the bow-shock of Vela X-1 is relatively bright. For comparison, the integrated flux of the "text-book-example" bow-shock around $\alpha$ Cam (distance $1.1 \mathrm{kpc}$ ) is $0.8,45,310$, and $470 \mathrm{Jy}$ (Van Buren et al. 1995) ${ }^{1}$. The angular extent of the bow-shock around

\footnotetext{
1 The latter values indicate that the dust in the bow-shock around Vela X-1 is hotter than that around $\alpha$ Cam. The X-ray source could provide additional heating.
} 
Vela $\mathrm{X}-1$ is $\sim 8^{\prime}$; at a 3 times larger distance it would just be resolvable in a HIRES image. Given the strong variations in the galactic infrared background emission, it is expected that several bow-shocks have remained undetected in the IRAS survey. However, bow-shocks as bright as those around Vela X-1 and $\alpha$ Cam should be detectable up to several $\mathrm{kpc}$.

A striking feature of Fig. 6 is the anisotropy of bowshock producing objects. A high concentration of bowshocks and IR-excess objects is found within $2 \mathrm{kpc}$ from the Sun in quadrant II $\left(90^{\circ} \leq l \leq 180^{\circ}\right)$, while in the opposite quadrant IV $\left(270^{\circ} \leq l \leq 360^{\circ}\right)$ the lowest fraction of bow-shocks is detected. The dumbell shape apparent in the contours of the bottom panel of Fig. 6 reflects the spiral-arm structure of the Milky Way.

\subsubsection{Space velocities}

In Fig. 7 the space velocity and distance $z$ with respect to the galactic plane are plotted of the OB runaways in our sample. This figure shows that most bow-shocks are detected around objects with velocities below $40 \mathrm{~km} \mathrm{~s}^{-1}$. Of the 76 objects that have a space velocity less than $40 \mathrm{~km} \mathrm{~s}^{-1}, 14$ are associated with a bow-shock and 18 show an IR-excess, together making up for about $42 \%$ of the total, while among the 72 objects that are faster than $40 \mathrm{~km} \mathrm{~s}^{-1}$ a lower fraction is found (6 bow-shocks and 10 objects with IR-excess, resulting in a $22 \%$ detection rate). At space velocities above $80 \mathrm{~km} \mathrm{~s}^{-1}$ none of the objects could be associated with a wind bow-shock, and only three (out of 18) were observed with excess infrared emission. Apparently, a very high space velocity is not a favourable condition for the production of a wind bow-shock. This observation is in agreement with the prediction on the basis of numerical simulations of bow-shocks by Comerón \& Kaper (1998) that at very high velocity a shockfront becomes unstable and disrupts.

Runaways with large separations from the galactic plane also show a low bow-shock detection rate. Among the objects that have a $z$ distance larger than 250 pc only 2 out of 13 were observed with an IR-excess (15\% detection rate). This low detection rate of bow-shocks at large galactic heights can be understood in terms of dust content. Since the dust content of the interstellar medium decreases with galactic height (Van Steenberg \& Shull 1988), a runaway too far from the galactic plane may not show an observable bow-shock because not enough dust is being swept up.

Both the space velocity and the height above the galactic plane are physical parameters that have to be taken into account when considering whether a bow-shock is observable or not. However, the majority of runaways without bow-shocks are not located at extreme distances from the galactic plane, nor have very high space velocities (Fig. 7). Obviously, other factors must play an important role as well.

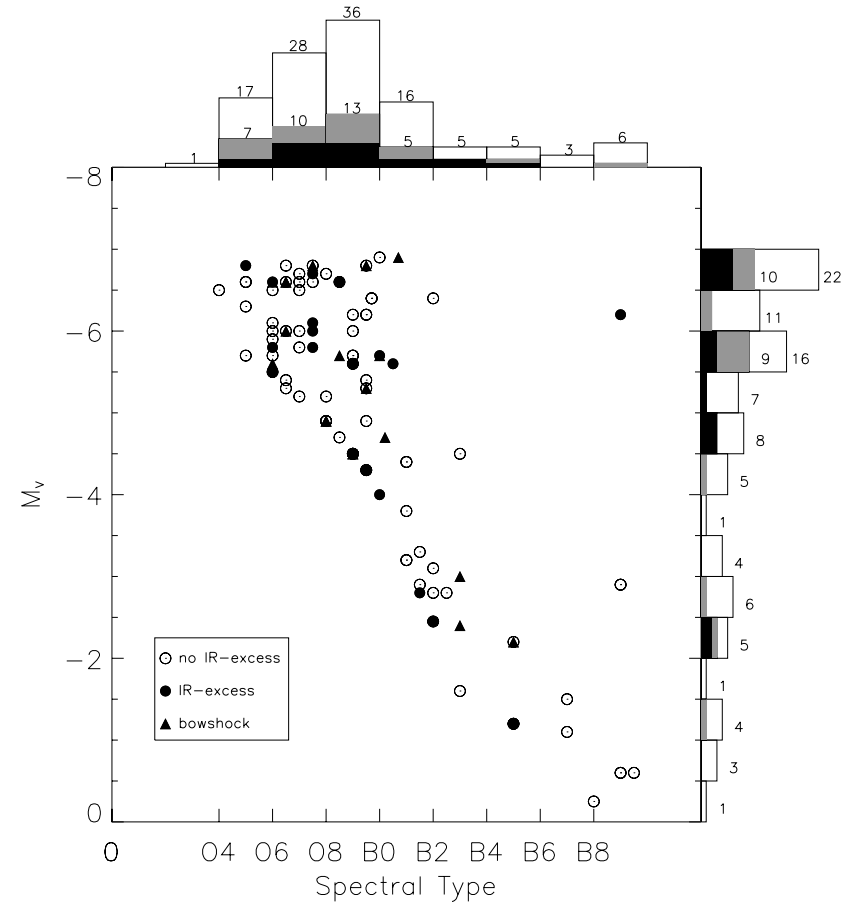

Fig. 8. Hertzsprung-Russell diagram of Van Buren's sample of runaways. Only objects located within $250 \mathrm{pc}$ from the galactic plane and with a space velocity in the range of 5 to $80 \mathrm{~km} \mathrm{~s}^{-1}$ are included. There is no obvious correlation between the detection of a bow-shock and the spectral type of the OB-runaway star.

\subsubsection{Dependence on spectral type}

Besides the space velocity of the OB-runaway star and the density of the ambient medium, the stellar-wind parameters $\dot{M}$ and $v_{\infty}$ determine the physical conditions under which a bow-shock is formed (cf. Eq. (1)). The wind mass-loss rate $\dot{M}$ is strongly dependent on the luminosity $\left(\dot{M} \propto L^{1.7}\right.$, Lamers \& Leitherer 1993; Puls et al. 1996), i.e. supergiants have much stronger winds than main sequence stars. Furthermore, the visibility of the wind bow-shock (at infrared wavelengths) depends on the irradiation of the swept-up dust by the ultraviolet flux from the massive star. One would expect that supergiants are more capable of forming a detectable bow-shock in comparison to main sequence stars.

In Fig. 8 the Hertzsprung-Russell diagram of the OB-runaway sample is presented; the symbols indicate whether a bow-shock (or IR excess) has been detected. The OB-runaway sample contains a relatively large fraction of supergiants and giants. This might be due to a selection effect: Hipparcos observed only the brightest stars on the sky. Inspection of the diagram learns that the detection rate of bow-shocks is about $35 \%$, independent of the luminosity class of the OB runaways:

- From a total of 36 supergiants (luminosity class I, Ia, Ib, Iab and II), six objects show bow-shocks and seven show excess infrared emission (36\% detection rate);

- The sample contains a total of 44 giants (luminosity class III and IV). Among these, seven show 
bow-shocks, and seven objects show excess infrared emission ( $32 \%$ detection rate);

- Out of 38 main sequence stars (luminosity class V), there are six with bow-shocks and eight with infrared excess (37\% detection rate).

Also the effective temperature of the star does not seem to affect the detectability of a wind bow-shock.

\section{On the absence of wind bow-shocks around runaway objects}

Although we found some observational indications that the presence of a wind bow-shock depends on the space velocity of the OB runaway and its height above the galactic plane, these findings cannot explain why the majority of systems do not produce a wind bow-shock. In the following we explore the possibility that perhaps some runaways are moving subsonically, despite their high space velocity. With space velocities found of several tens of $\mathrm{km} \mathrm{s}^{-1}$ this would require extreme conditions of the interstellar medium, conditions that are known to be present in hot bubbles and galactic coronal gas.

The (isothermal) speed of sound in the interstellar medium (ISM) has a value of $\sim 1 \mathrm{~km} \mathrm{~s}^{-1}$ at a temperature of $100 \mathrm{~K}, \sim 10 \mathrm{~km} \mathrm{~s}^{-1}$ at $10^{4} \mathrm{~K}$ and $\sim 100 \mathrm{~km} \mathrm{~s}^{-1}$ at $10^{6} \mathrm{~K}$. Runaway stars thus move supersonically unless they travel through regions with temperatures of the order of $10^{6} \mathrm{~K}$. Such hot regions are present in the Milky Way, the so-called hot bubbles, and appear e.g. as holes in the distribution of neutral interstellar matter (Heiles 1979, 1984, 1998; Shull \& Saken 1995). The hot regions are likely produced by the combined action of stellar winds and supernovae in a central star cluster (i.e. an OB association), which sweeps the surrounding gas and dust away and confines this material into dense filaments (walls). The ambient density of the medium is thereby reduced to values $\sim 10^{-3} \mathrm{~cm}^{-3}$. The temperature may reach $10^{6} \mathrm{~K}$, resulting in the production of diffuse X-ray emission, such as observed from the Cygnus superbubble (Cash et al. 1980). The ionized gas inside the hot bubble can also be observed at radio wavelengths, with a synchrotron component originating near the walls. The Sun is located in the local hot bubble, a hot medium with a deficiency of neutral matter. The walls of this bubble are fairly well defined by the distribution of interstellar dust (Lucke 1978) and the detection of interstellar absorption lines (Frisch \& York 1983). Recently, Heiles (1998) reported the discovery of a nearby superbubble which may have merged with the local bubble, forming a highly oval cavity elongated towards $l \sim 230$.

To test the hypothesis that such hot interstellar gas indeed prevents runaways from producing wind bow-shocks, we investigated the distribution of runaways in relation to the distribution of hot bubbles in the galactic plane. We used the H I surveys carried out by Heiles (1979, 1984), and compiled a list of $\mathrm{H}$ I shells, supershells and other shell-like objects. The surveys span a range in galactic

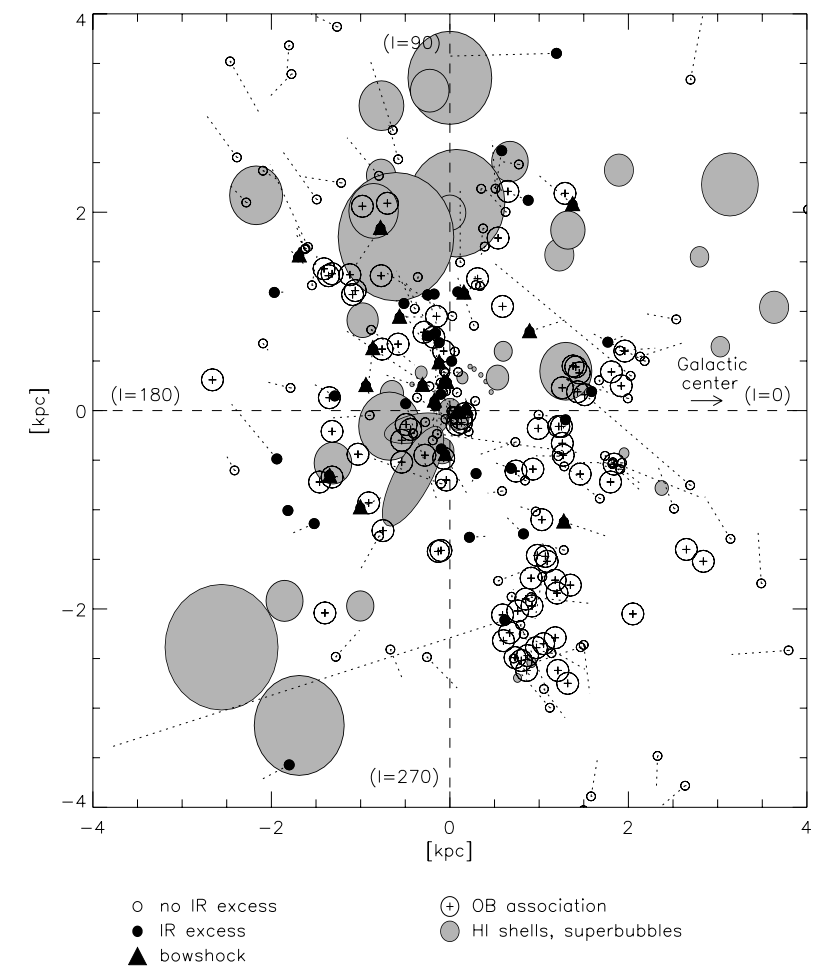

Fig. 9. The distribution of $\mathrm{H}$ I shells in the galactic plane, in the range $10 \leq l \leq 250$ and $-10 \leq b \leq 10$ according to Heiles $(1979,1987)$ (shaded regions). The fourth quadrant of the galactic plane has not been included in these studies. The Sun is located in the origin of the diagram; the direction of the galactic center is indicated. OB associations (taken from Melnik \& Efremov 1995) are represented by circles including a plus sign (see legenda). OB runaways and their paths (the path length corresponds to the projected distance travelled during the last $10 \mathrm{Myr}$ ). The large concentration of OB associations in the fourth quadrant suggests that also here hot bubbles must be present. Most of the OB runaways producing a bow-shock do not seem to be contained inside a hot bubble.

longitude $10^{\circ} \leq l \leq 250^{\circ}$ and latitude $-10^{\circ} \leq b \leq 10^{\circ}$. The recently discovered nearby superbubble (Heiles 1998), next to the Local Bubble, is also included. The studies by Heiles do not cover the fourth quadrant of the galaxy $\left(270^{\circ} \leq l \leq 360^{\circ}\right)$. The large number of OB associations in that area make it very likely that hot bubbles are present there as well. At longitudes $317^{\circ}$ and $327^{\circ}$ filaments extending over several degrees are detected in the Australian Mononglo (MOST) survey (Green et al. 1999).

Figures 9 and 10 show the locations and trajectories of runaways (the length of the track corresponds to the distance travelled in $10 \mathrm{Myr}$ ), projected onto the galactic plane, with respect to $\mathrm{H}$ I shells and bubbles. Figure 10 (a zoom in on the central region in Fig. 9), focusses on the solar neighbourhood (within $1.3 \mathrm{kpc}$ ). OB associations are represented as circles with a cross in the middle (see legenda); in Fig. 10 the associations comprised in De Zeeuw et al. (1999) are included. Apart from Heiles' New Superbubble and the Eridanus Loop, the shape of the $\mathrm{H}$ I shells is assumed to be spherically symmetric. The estimated distance and angular size in galactic latitude and 


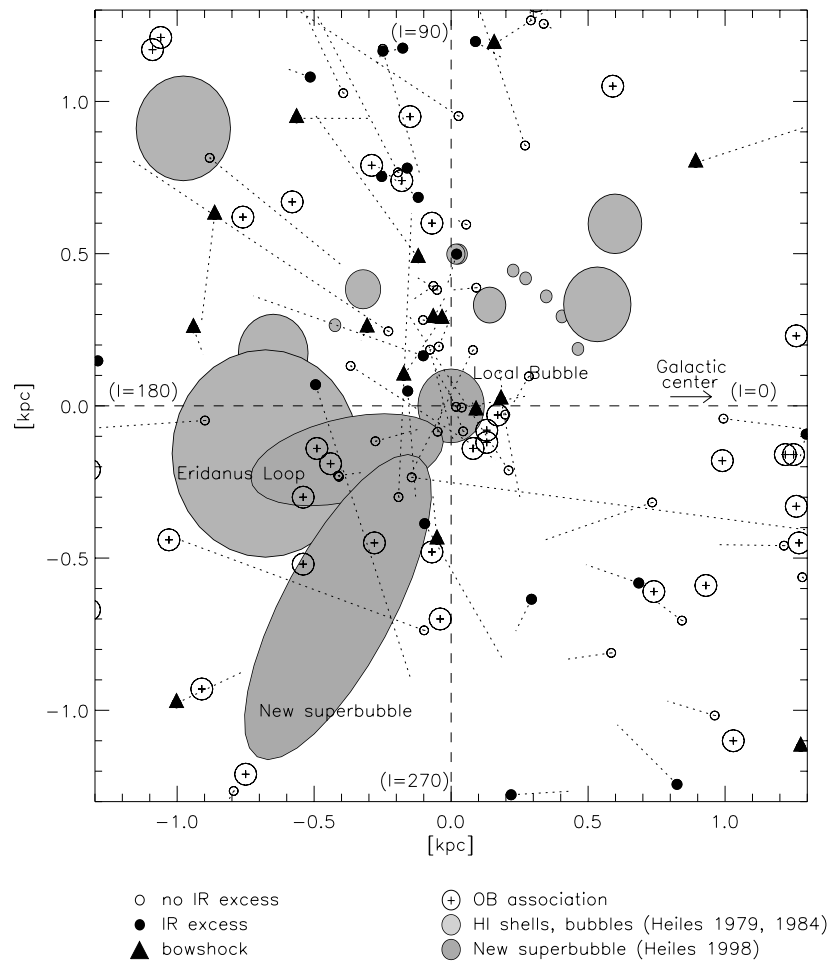

Fig. 10. Zoom-in on the central area of Fig. 9. Some wellknown hot bubbles are labelled.

longitude are used to determine the radius of the bubble. A large degree of uncertainty exists in the size of the bubbles along the line of sight.

Although both the OB-runaway and the hot-bubble distribution are not accurately known, the OB runaways producing bow-shocks are mostly found in regions devoid of hot bubbles. Remarkable is the asymmetry in the distribution of $\mathrm{OB}$ runaways producing bow-shocks with respect to the Sun: quadrant II (in the direction of Cepheus) contains significantly more bow-shocks than the other quadrants. This anisotropy agrees with the distribution of hot bubbles. In the nearby (super)bubbles we hardly encounter any OB runaway with a bow-shock.

Dove \& Shull (1994) estimate that the average volume filling factor of (super)bubbles and cavities in the galactic plane is $50-80 \%$, which is consistent with the fraction of bow-shock producing objects among the runaway stars in Van Buren's sample. Apparently, the soundspeed in these regions is high enough to prevent the formation of wind bow-shocks.

\subsection{The $H M X B$ sample}

Can the detection of a wind bow-shock around only one HMXB (out of 11) also be explained by the distribution of hot bubbles? Figure 11 displays the distribution of the HMXBs in our sample. Cyg X-1 is located close to the edge of the Cygnus superbubble, while Vela X-1, the only HMXB for which a bow-shock clearly has been detected, seems to move through a medium devoid of hot bubbles.

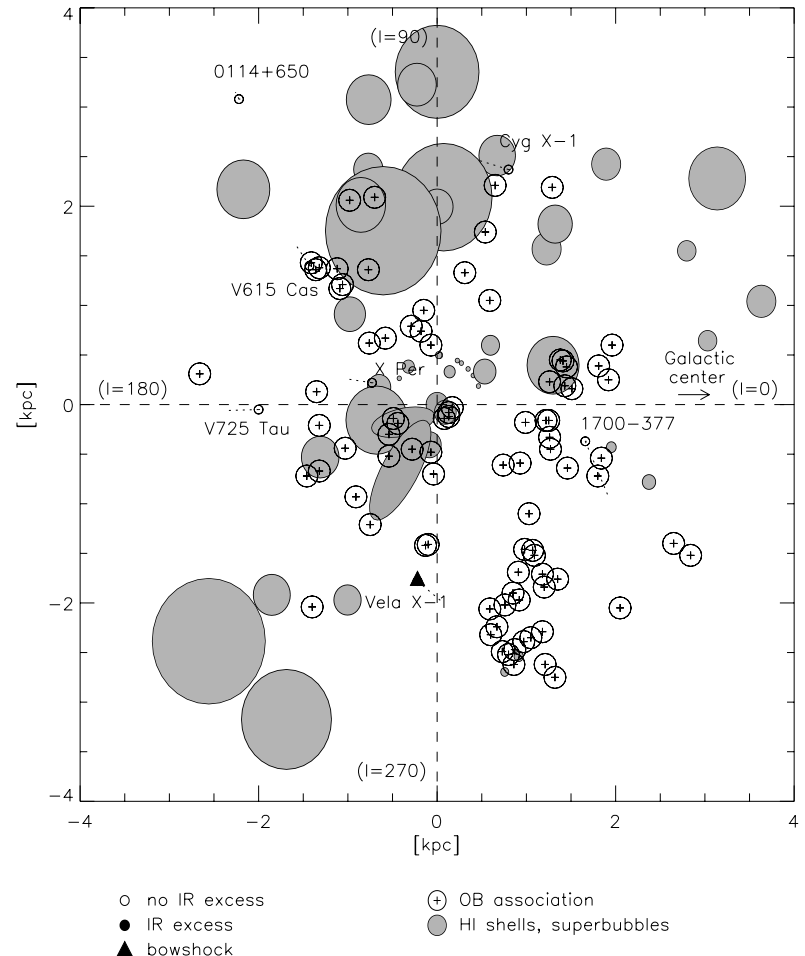

Fig. 11. The distribution of the HMXBs in our sample is shown with respect to hot bubbles and OB associations. Each HMXB shows a track that reflects the distance travelled in $10 \mathrm{Myr}$; in some cases these tracks identify the parent $\mathrm{OB}$ association of the system. Cyg X-1 and X Per are very likely enclosed in hot superbubbles. Also 1700-377 and V615 Cas may be situated in such hot regions, although the presence of bubbles at the positions of 1700-377 and V615 Cas is uncertain.

At least four HMXBs are likely to be travelling through a hot bubble.

1700-377. Benaglia \& Cappa (1999) report the presence of an $\mathrm{H}$ I structure that may be due to a wind-blown bubble created by the O-supergiant companion of 1700377, HD 153919. The H I region increases in size towards higher galactic latitudes, forming some kind of horseshoe shape. The mean velocity of the bubble corresponds to a distance $\sim 2 \mathrm{kpc}$, in agreement with the distance of 1700377 (cf. Ankay et al. 2001).

V615 Cas. According to Fig. 11, this HMXB is located near the edge of a large superbubble; two other bubbles are found at roughly the same longitude. Shell GS $135+08-34$ has a larger catalogued distance than V615 Cas (3.1 and $2.0 \mathrm{kpc}$, respectively), but taking into account the uncertainty in these distance determinations, they could possibly be associated with each other. On the other hand, the center of GS $135+08-34$ lies at a larger angular separation from the galactic plane than V615 Cas $\left(+8^{\circ}\right.$ as compared to $\left.+1^{\circ}\right)$. It is not quite clear whether the extent of GS $135+08-34$ is sufficient to enclose V615 Cas. Heiles (1984) estimates an angular size of only $\sim 6^{\circ}$ of GS $135+08-34$ in galactic latitude.

X Per. Two H I shells can be discerned in the vicinity of X Per. The smaller of the two bubbles (GS 165-21-4) is 


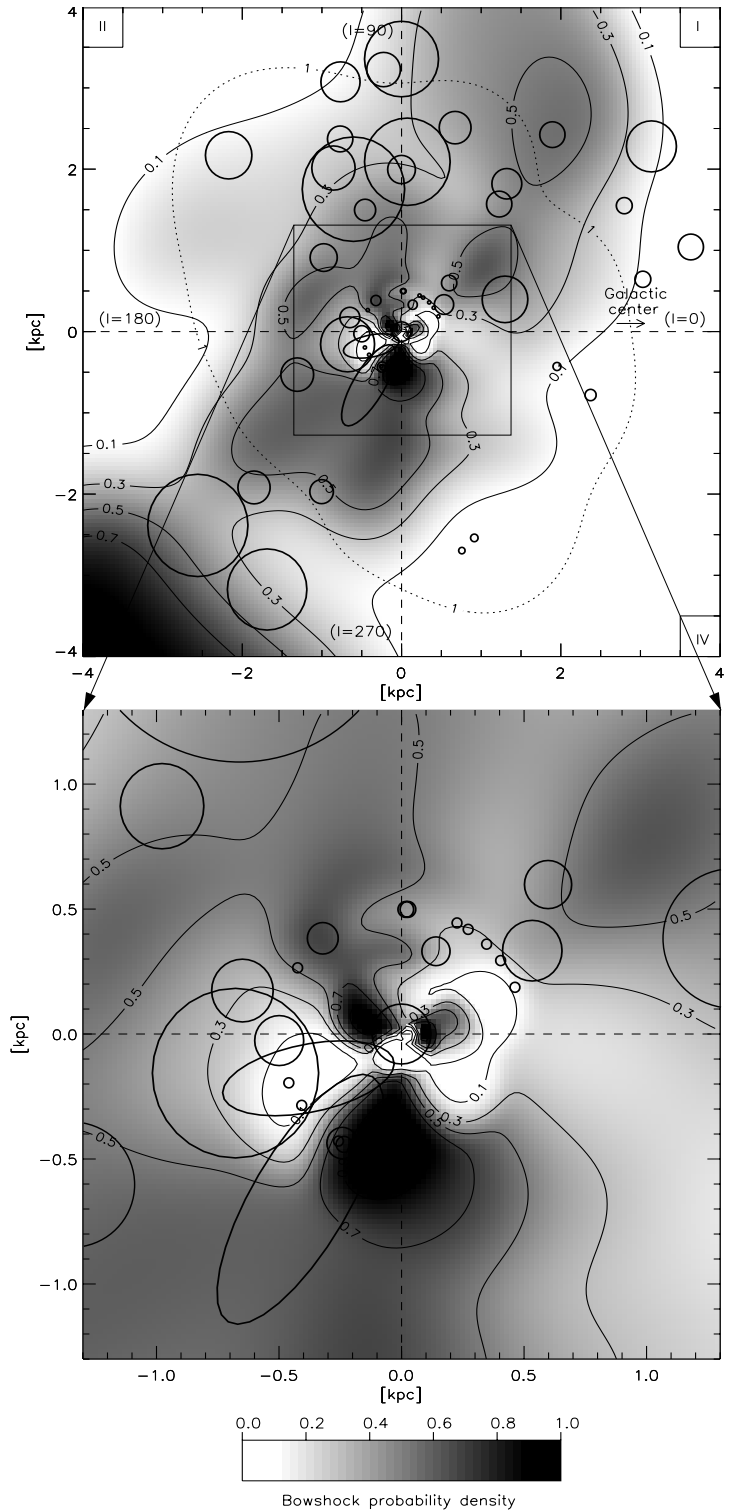

Fig. 12. The fraction of bow-shock producing runaways ("bowshock probability density") is shown together with the observed distribution of local hot bubbles. The bow-shock probability distribution is determined with objects that are located within $300 \mathrm{pc}$ from the galactic plane and have space velocities between 5 and $80 \mathrm{~km} \mathrm{~s}^{-1}$. The HMXB sample is also included, yielding a total sample of 137 runaways. In the top panel, the dotted contour follows a runaway number density of $1 \mathrm{kpc}^{-2}$, marking the region outside of which the bow-shock probability density is determined by less than 1 object per square kpc.

centered around galactic latitude $b=-21^{\circ}$ corresponding to a separation from the galactic plane of $\sim 260 \mathrm{pc}$ at a distance of $0.72 \mathrm{kpc}$ (Heiles 1984). The extent of this shell along the $z$-direction is estimated to be $\sim 160$ pc at that distance. X Per is situated 235 pc below the galactic plane, and is therefore likely enclosed in this bubble.

Cyg X-1. This HMXB is located in the boundary region of the Cygnus superbubble (see Fig. 2). Cash et al. (1980) performed observations with HEAO-1 of this region and showed that the observed X-rays emitted from this region is outlined by a ring of elongated $\mathrm{H} \alpha$ filaments, suggesting a common origin. Moreover, interstellar absorption considerations yield a distance of about $2 \mathrm{kpc}$ of the $\mathrm{X}$ ray feature, thus at roughly the same distance as Cyg X-1. The temperature of the X-ray emitting plasma contained in the Cygnus superbubble is estimated to be $2 \times 10^{6} \mathrm{~K}$.

\section{Discussion}

One of the main conclusions of our work is that the formation of a wind bow-shock around an OB-runaway star strongly depends on the temperature and density of the ambient medium. Apparently, the majority of the OB runaways are moving through hot bubbles distributed in the galactic plane. These hot bubbles are created by the combined action of stellar winds and supernovae of OB stars, most of them members of OB associations. Assuming that all $\mathrm{OB}$ runaways originate in $\mathrm{OB}$ associations, it is very likely that they travel through such hot bubbles for a substantial fraction of their (relatively short) lifetime. In the following we use the absence of a wind bow-shock as a diagnostic to probe the distribution of hot bubbles in the solar neighbourhood. Finally, we present arguments why HMXBs should have a higher probability to be located inside a hot bubble than dynamically ejected OB runaways.

\subsection{The distribution of hot bubbles in the solar neighbourhood}

Various people have tried to map out the extent of the local bubble (see Frisch 1995 and references therein). Due to its high temperature, some nearby runaways that are moving through this bubble are not producing wind bowshocks, even though they have substantial space velocities. The presence, or rather the absence, of bow-shocks can thus serve as a diagnostic to constrain the extent of the local bubble. An attempt to map out hot galactic regions based on the relative presence of bow-shock producing runaways is shown in Fig. 12. The bow-shock probability density reflects the fraction of runaways producing a bowshock. The galactic distribution is derived using the binormal kernel method (adopting $\sigma_{r}=0.30 r$, cf. Sect. 3.1.1); the spatial distribution of OB runaways producing bowshocks (top panel Fig. 6) is divided by the spatial distribution of the whole sample of OB runaways, including the HMXBs (bottom panel Fig. 6). Some OB runaways can be expected to not produce a bow-shock, because of their high space velocity or their large separation from the galactic plane. For that reason, the objects used for determining the probability distribution in Fig. 12 are only those with intermediate velocities $\left(5 \mathrm{~km} \mathrm{~s}^{-1} \leq V_{\text {space }} \leq\right.$ $80 \mathrm{~km} \mathrm{~s}^{-1}$ ), and those that are located within $300 \mathrm{pc}$ of the galactic plane. Note that areas with a small number of runaways will have a rather inaccurate value of the bowshock probability density (division through a small number). Figure 13 illustrates that the obtained distribution is not very sensitive to the value of $a$; for $\sigma_{\mathrm{r}}=0.5 r$ the 
space distribution becomes uniform at distances beyond $\sim 0.7 \mathrm{kpc}$ from the Sun.

It can be seen that the distribution of nearby hot bubbles (from $\mathrm{H} 1$ measurements) is consistent with the distribution of regions showing a low fraction of bow-shock producing runaways. At larger distances this method of mapping hot regions becomes inaccurate due to the rapidly decreasing number of objects. For example, the probability density maximum in the lower left corner of Fig. 12 (top panel) is completely determined by only one object beyond $3 \mathrm{kpc}$ in that quadrant, an object that happens to show excess infrared emission.

\section{2. $H M X B$ s have a lower probability to produce bow-shocks than dynamically ejected $O B$ runaways}

Only one of the 11 HMXBs has an associated wind bowshock. Though small-number statistics, does this indicate that HMXBs have a lower probability to form a wind bowshock? The bow-shock detection rate for Van Buren's sample is about $40 \%$. If we take into account that the detection sensitivity of IRAS is poor for objects beyond $2 \mathrm{kpc}$, then we are left with 1 out of 5 HMXBs. These numbers do not provide statistically significant results, but the different evolutionary origin, and therefore on average different kinematical age, of HMXBs and dynamically ejected runaways might result in a difference in detection rate of wind bow-shocks.

Massive stars ejected by dynamical interactions should have escaped the $\mathrm{OB}$ association at a relatively early stage, when the cluster is still dense and the probability for close encounters high. On the other hand, OB stars in HMXBs went through several stages of binary evolution before they became runaways after the occurence of a supernova within the system. Therefore, the kinematical age (i.e. the travel time since ejection took place) of HMXBs should be smaller than those of dynamically ejected runaways. As a consequence, HMXBs have a higher probability to be still enclosed in the hot and rarefied regions (e.g. superbubbles) that surround $\mathrm{OB}$ associations. Further study is required to prove this hypothesis with observations.

\section{Summary and conclusions}

Based on our study of OB runaways and the phenomenon of wind-bow-shock formation we conclude that about $40 \%$ of the OB-runaway stars produce wind bow-shocks. This fraction is a little bit higher than proposed by Van Buren and coworkers, but takes into account the estimated reduction in IRAS sensitivity required to detect a bow-shock. We investigate why the majority of OB runaways do not produce a bow-shock. The spectral type (temperature, luminosity, mass-loss rate), space velocity and separation from the galactic plane seem to be of minor importance. We propose that the temperature and density of the ambient medium are the dominant factors. In hot bubbles

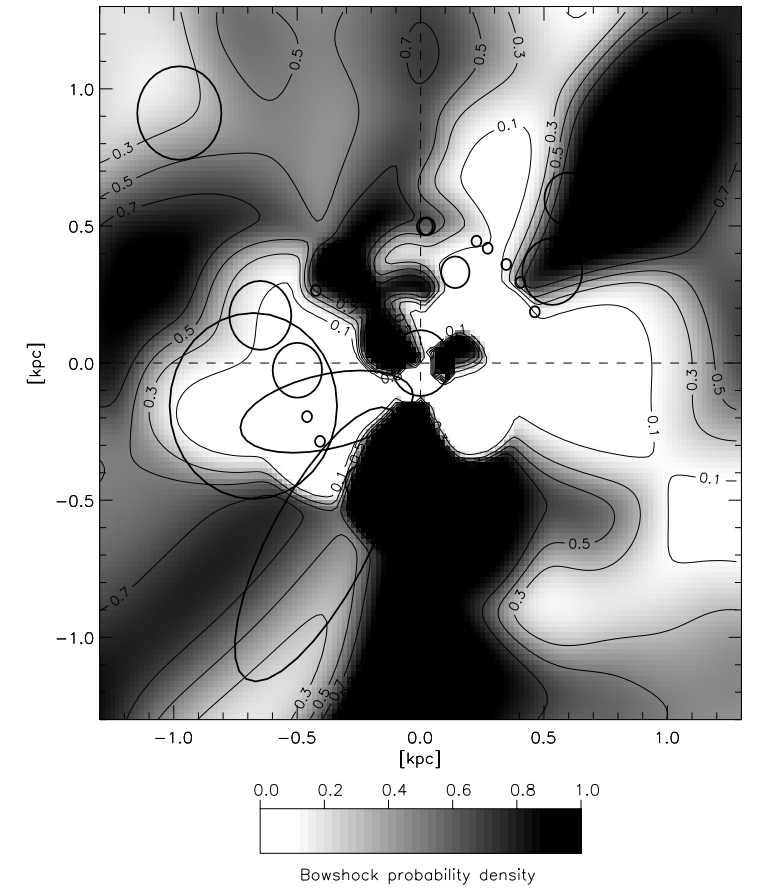

Fig. 13. As the bottom panel of Fig. 12, but now calculated adopting $\sigma_{r}=0.15 r$ to illustrate that the obtained space distribution is not very sensitive to the value of $a$.

the speed of sound is larger than the typical velocity of a runaway star $\left(\sim 50 \mathrm{~km} \mathrm{~s}^{-1}\right)$, so that runaways in these regions do not move supersonically and would not produce a bow-shock. OB-runaways produced through the binary supernova scenario should on average have a shorter kinematical age than those produced through the cluster ejection scenario, and thus have a higher probability to be still inside a hot bubble. For some runaways, e.g. Cyg X-1, there is observational evidence that the system indeed is contained in a (super)bubble.

Acknowledgements. We are grateful to Romke Bontekoe and Do Kester for the permission to work with their software, and their help and advice. Ben Stappers, Ed van den Heuvel, and the referee are thanked for a careful reading of the manuscript and useful suggestions. The IRAS data were obtained using the IRAS data base server of the Space Research Organisation of the Netherlands (SRON) and the Dutch Expertise Centre for Astronomical Data Processing funded by the Netherlands Organisation for Scientific Research (NWO). The IRAS data base server project was also partly funded through the Air Force Office of Scientific Research, grants AFOSR 86-0140 and AFOSR 89-0320. We acknowledge the use of the SIMBAD database. LK is supported by a fellowship of the Royal Netherlands Academy of Arts and Sciences.

\section{References}

Ankay, A., Kaper, L., De Bruijne, J. H. J., et al. 2001, A\&A, 370,170

Baranov, V. B., Krasnobaev, K. V., \& Kulikovskii, A. G. 1971, Sov. Phys. Dokl., 15, 791 
Benaglia, P., \& Cappa, C. E. 1999, A\&A, 346, 979

Blaauw, A. 1961, Bull. Astr. Inst. Netherlands, 15, 265

Blaauw, A. 1964, Ann. Rev. Astr. Ap., 2, 213

Blaauw, A. 1978, in Problems of Physics and Evolution of the Universe, ed. L. Mirzoyan (Yerevan USSR), 101

Blaauw, A. 1993, in Massive Stars: their lives in the ISM, ed. J. P. Cassinelli \& E. B. Churchwell, ASP Conf. Ser., 35, 207

Boersma, J. 1961, Bull. Astr. Inst. Netherlands, 505, 265

Bontekoe, Tj. R., Koper, E., \& Kester, D. J. M. 1994, A\&A, 284, 1037

Cash, W., Charles, P., Bowyer, S., et al. 1980, ApJ, 238, L71

Chevalier, C., \& Ilovaisky, S. A. 1998, A\&A, 330, 201

Comerón, F., \& Kaper, L. 1998, A\&A, 338, 273

Conlon, E. S., Dufton, P. L., Keenan, F. P., \& Leonard, P. J. T. 1990, A\&A, 236, 357

Cruz-Gonzalez, C., Recillas-Cruz, E., Costero, R., et al. 1974, Rev. Mexicana Astr. Ap., 1, 211

De Zeeuw, P. T., Hoogerwerf, R., De Bruijne, J. H. J., et al. 1999, AJ, 117, 354

Dickey, J. M., \& Lockman F. J. 1990, ARA\&A, 28, 215

Dove, J. B., \& Shull, J. M. 1994, ApJ, 430, 222

Draine, B., \& Lee, H. M. 1985, ApJ, 285, 89

Feast, M. W., \& Shuttleworth, M. 1965, MNRAS, 130, 245

Fowler, J. W., \& Aumann, H. H. 1994, in Science with High Spatial Resolution Far-Infrared Data, ed. S. Terebey, \& J. Mazzarella, 1

Frisch, P. C., \& York, D. G. 1983, ApJ, 271, L59

Frisch, P. C. 1995, Space Sci. Rev., 72, 499

Garmany, C. D., Conti, P. S., \& Chiosi, C. 1982, ApJ, 263, 777

Gies, D. R., \& Bolton, C. T. 1986, ApJS, 61, 419

Green, A. J., Cram, L. E., Large, M. I., et al. 1999, ApJS, 122, 207

Heiles, C. 1979, ApJ, 229, 533

Heiles, C. 1984, ApJ, 55, 585

Heiles, C. 1987, ApJ, 315, 555

Heiles, C. 1998, ApJ, 498, 689

Hoogerwerf, R., De Bruijne, J. H. J., \& De Zeeuw, P. T. 2001, A\&A, 365, 49

Kaper, L. 1998, in Proc. Boulder-Munich II: Properties of Hot, Luminous Stars, ed. I. D. Howarth, ASP Conf. Ser., 131, 427
Kaper, L. 2001, in Proc. The influence of binaries on stellarpopulation studies, ed. D. Vanbeveren (Kluwer Acad. Pub.), 125

Kaper, L., Van Loon, J. Th., Augusteijn, T., et al. 1997, ApJ, 475, L37

Kaper, L., Cómeron, F., \& Barziv, O. 1999, in Wolf-Rayet Phenomena in Massive Stars and Starburst Galaxies, ed. K. A. van der Hucht, G. Koenigsberger, \& P. R. J. Eenens, ASP Conf. Ser., 193, 316

Lamers, H. J. G. L. M., \& Leitherer, C. 1993, ApJ, 412, 771

Leonard, P. J. T. 1995, MNRAS, 227, 1080

Lucke, P. B. 1978, A\&A, 64, 367

McKee, C. F., \& Ostriker, J. P. 1977, ApJ, 218, 148

Melnik, A. M., \& Efremov, Y. N. 1995, Pis'ma Astron. Zh., 21, 13

Mikkola, S. 1983, MNRAS, 205, 733

Moffat, A. F. J., Marchenko, S. V., Seggewiss, W., et al. 1998, A\&A, 331, 949 (erratum)

Moshir, H., Kopan, G., Conrow, T., et al. 1989, Explanatory Supplement to the IRAS faint source survey, IPAC

Noriega-Crespo, A., Van Buren, D., \& Dgani, R. 1997, AJ 113, 780

Portegies Zwart, S. F. 2000, ApJ, 544, 437

Poveda, A., Ruiz, J., \& Allen, C. 1967, Bol. Obs. Tonantzintla y Tacubaya, 4,86

Puls, J., Kudritzki, R. P., Herrero, A., et al. 1996, A\&A, 305, 171

Shull, J. M., \& Saken, J. M. 1995, ApJ, 444, 663

Stone, R. C. 1979, ApJ, 232, 520

Van Buren, D., \& McCray, R. 1988, ApJ, 329, L93

Van Buren, D., Noriega-Crespo, A., \& Dgani, R. 1995, AJ, 110 2914

Van den Heuvel, E. P. J., \& Heise, H. 1972, Nature Phys. Sci., 239,67

Van den Heuvel, E. P. J., Portegies Zwart, S. F., Bhattacharya, D., \& Kaper, L. 2000, A\&A, 364, 563

Van Oijen, J. G. J. 1987, A\&A, 217, 115

Van Steenberg, M. E., \& Shull, J. M. 1988, ApJ, 335, 197

Wilkin, F. P. 1996, ApJ, 459, L31

Zwicky, F. 1957, Morphological Astronomy (Springer, Berlin), 258 